\title{
Bone Mineral Density as a Potential Predictive Factor for Luminal-type Breast Cancer in Postmenopausal Women
}

\author{
FRANCO LUMACHI ${ }^{1}$, STEFANO M.M. BASSO ${ }^{2}$, VALENTINA CAMOZZI ${ }^{3}$, \\ ROBERTO SPAZIANTE ${ }^{4}$, PAOLO UBIALI ${ }^{2}$ and MARIO ERMANI ${ }^{5}$ \\ ${ }^{1}$ Department of Surgery, Oncology \& Gastroenterology, School of Medicine, University of Padua, Padua, Italy; \\ ${ }^{2}$ Department of Surgery, S. Maria degli Angeli Hospital, Pordenone, Italy; \\ ${ }^{3}$ Department of Medicine (DiMed), School of Medicine, University of Padua, Padua, Italy; \\ ${ }^{4}$ Department of Diagnostic Imaging, S. Maria degli Angeli Hospital, Pordenone, Italy; \\ ${ }^{5}$ Department of Neurosciences (DNS), Statistic and Informatics Unit, \\ School of Medicine, University of Padua, Padua, Italy
}

\begin{abstract}
Background/Aim: We performed a case-control study to evaluate whether bone mineral density $(B M D)$ can be considered a potential predictive factor for luminal-type breast cancer $(B C)$, that could be useful in constructing a predictive risk model. Materials and Methods: The medical records of 297 postmenopausal women with luminal-type node-negative $B C$ who underwent lumbar-spine dual-energy $X$-ray absorptiometry (DXA) with BMD measurement before surgery, were analyzed and compared with those of 297 age-matched randomly selected healthy controls. The correlations between women's reproductive history, including the age at menarche and menopause, parity, oral contraceptives and hormone replacement therapy (HRT) use, the results of DXA, and BC risk were evaluated in univariate and multivariate analyses. Results: Overall, 168 (28.3\%) women had osteoporosis and/or osteopenia (low BMD). Both bone alterations were protective factors for $B C$, especially when they were considered together $(p=0.001)$. Only the interval between menarche and menopause (MMI), dichotomized at 37.5 years as an optimal cut-off, and the HRT use reached a statistical significance
\end{abstract}

This study was presented in part at the World Congress on Osteoporosis, Osteoarthritis and Musculoskeletal Diseases (WCO), International Osteoporosis and Other Skeletal Diseases Foundation (IOF), European Society for Clinical and Economical Aspects of Osteoporosis, Osteoarthritis and Musculoskeletal Diseases (ESCEO) Joint Meeting, Malaga (Spain), April 14-17, 2016.

Correspondence to: Dr. Stefano Maria Massimiliano Basso, Department of Surgery, "S. Maria degli Angeli" Hospital, via Montereale 24, 33170 Pordenone, Italy. Tel: +39 0434 399359, Fax: +390434 399704, e-mail: Drsteba@tin.it

Key Words: Breast cancer, luminal-type, menopause, BMD, osteoporosis, predictive factors. $(p<0.01)$ as risk factors. The three parameters were independent because they remained significant in the stepwise logistic regression analysis. The area under the receiver operating characteristic (ROC) curve (AUC) obtained with the model was 0.694 (95\%CI=0.694-0.731). Conclusion: This hypothesized predictive model is fairly accurate and could identify patients at increased risk of developing luminal-type $B C$ in a population of postmenopausal women who performed DXA, simply based on their history.

Breast cancer (BC) and bone mineral loss, including osteopenia and osteoporosis, are both significant global health problems among postmenopausal women, especially in Western countries $(1,2)$. Breast cancer is an extremely heterogeneous disease, and $\mathrm{BC}$ incidence shows several geographical disparities, ranging from approximately 50 to 150 cases per 100,000 people per year $(3,4)$. It has been estimated that up to $30-35 \%$ of postmenopausal women have osteoporosis (5). The relationship between $\mathrm{BC}$ and bone mineral density (BMD) has since long been investigated because both mainly depend on lifelong exposure to endogenous estrogens, which chronically stimulate both mammary tissue and trabecular bone (6). According to a recent systematic review, high BMD in the lumbar spine (LS) is associated with a 1.82 relative risk of having BC, corresponding to an increased risk of approximately $80 \%$ (7). A number of modifiable and non-modifiable risk factors (RFs) for BC, other than high BMD, have been identified, including lifestyle (e.g., high fat and alcohol consumption, low physical activity, overweight and obesity), personal or family history of cancer, reproductive history (e.g., age at menarche and menopause, pregnancies, breastfeeding), and the use of oral contraceptives and hormone replacement therapy (HRT) (8-10). Protective factors (PFs), including dietary and nutritional adjustments, parity, and the use of 
antiestrogen drugs, are less frequently investigated (11). Unfortunately, the majority of such factors, including BMD alterations, are strictly interdependent; thus, the real effect of each factor is difficult to assess.

The aim of this study was to evaluate whether the presence of osteopenia or osteoporosis (low BMD) can be considered an independent $\mathrm{PF}$ for luminal-type $\mathrm{BC}$ in postmenopausal women, which could be useful in constructing a simple predictive risk model.

\section{Materials and Methods}

Study population. According to the self-reported age at the last menstruation, spontaneous menopause was defined as the end of menses for at least 12 months (6). With the aim of having a study population as homogeneous as possible, 297 medical records (cases) of postmenopausal women with pT1-2, node-negative (pN0), estrogen receptor (ER)-positive (luminal-type), HER2-negative invasive ductal carcinomas were obtained from our cumulative preexisting databank of patients who underwent curative surgery for BC. The final diagnosis and the intrinsic subtype profile were obtained by routine histology and immunohistochemical methods, performed on surgical specimens. The pT staging was the following: pTis=7 (2.4\%), pT1a=18 (6.1\%), pT1c=151 (50.8\%), pT2=121 $(40.7 \%)$; the AJCC anatomic stages were 0 , IA, and IIA. To exclude any possible confounding data and potential bias, patients with other histologic types of BC were considered not suitable for the study, as were those who had non-luminal type (ER-negative) or node-positive (N1-3) BC, had a HER2-positive BC, or required neoadjuvant chemotherapy. Other exclusion criteria were: (i) a history of previous cancer, hysterectomy or bilateral oophorectomy before their cancer diagnosis, chronic diseases (e.g., diabetes mellitus), abnormal dietary regimens (e.g., excessive fat intake), alcohol abuse or current smoking; (ii) excessive vitamin D and/or calcium intake, or the use of drugs potentially able to modify BMD, including bisphosphonates, calcitonin, teriparatide, and denosumab. All patients underwent lumbar spine (L2-L4) dual-energy X-ray absorptiometry (DXA) with BMD measurement (Hologic QDR $4500 \mathrm{C}$, Waltham, MA, USA), approximately from one to three months before surgery. In vitro, the coefficient of variation $(\mathrm{CV})$ of DXA was $0.6 \%$ (calculated by performing 20 scans of the Hologic Anthropometric Spine Phantom) and in vivo, the $\mathrm{CV}$ was $1.2 \%$ (calculated performing two lumbar scans in 10 healthy volunteers), as previously reported (12).

The controls were 297 age-matched healthy postmenopausal women, who were randomly selected from the databank of patients who performed DXA with the same instrument and software and met the same exclusion criteria as the cases. The controls have undergone annual screening mammography twice, to virtually exclude the presence of a subclinical BC.

In the overall population $(\mathrm{N}=594)$, the following main estrogenrelated parameters were recorded during the pre-DXA interview: the age at menarche and menopause, parity, the age at first pregnancy, breast-feeding, body mass index (BMI, $\mathrm{kg} / \mathrm{cm}^{2}$ ), and oral contraceptives or HRT use. According to the WHO guidelines, osteopenia was defined as a BMD between 1.0 and 2.5 standard deviations (SD) below that of a young adult (T-score between -1.0 and $-2.5 \mathrm{SD}$ ), and osteoporosis was defined as a T score of $-2.5 \mathrm{SD}$ or lower $(13,14)$. Serum estradiol measurement, obtained from 10 to 20 days before DXA, was available for all patients.
Statistical analysis. The continuous data were expressed as the mean \pm SD or standard error (SE). Two-tailed Student's $t$-test for unpaired data and the chi-square $\left(\chi^{2}\right)$ test and contingency tables were used for inter-group comparisons of the means and dichotomous variables, respectively. Odds ratio (OR) estimates and associated $95 \%$ confidence intervals (CI) for matching case-controls were used as measures of association. The sensitivity, specificity, positive predictive value, negative predictive value, and the likelihood ratio (LR) were also calculated. The parameters (independent variables) found to be significantly related to $\mathrm{BC}$ (dependent variable) were assessed for the multivariate analysis using a forward stepwise logistic regression model. The parametric statistical Wald test and the Youden method were used to compare the differences between the maximum LRs, and to identify the optimal cut-off risk of dichotomous variables, respectively. The receiver operating characteristic (ROC) curve was used to represent the diagnostic accuracy, and the area under the curve (AUC) was obtained. A $p$-value of 0.05 or less was considered significant for all analyses. Statistical calculations were performed with the Statistica software (StatSoft, Tulsa, OK, USA, version 2012).

\section{Results}

Overall, $168(28.3 \%)$ women had low BMD (osteoporosis and/or osteopenia), while 426 (71.7\%) had normal BMD for their age. The main characteristics of the cases and controls and the relative $p$-value estimations are reported in Table I. The interval between menarche and menopause (MMI) was significantly longer in patients with BC, who also used HRT for a longer time. Serum estradiol and the other reported continuous parameters, including family history of $\mathrm{BC}$ $(p=0.09)$, alcohol abuse $(p=0.44)$, and smoking $(p=0.67)$, did not differ significantly. Table II displays the distribution of the main estrogen-related dichotomous parameters, showing that only the use of HRT and low BMD were significantly $(p<0.05)$ different between groups.

The results of the univariate analysis using MMI (dichotomized at $\geq 37.5$ years), HRT, and normal BMD as risk factors for $\mathrm{BC}$ are reported in Table III.

In the multivariate analysis, MMI and HRT were RFs, while the presence of low BMD was a PF (Table IV). In addition, the age, as well as the MMI, should not be considered as a linear variable, and thus it was dichotomized using 55.5 years as the optimal cut-off. The new results obtained are reported in Table $\mathrm{V}$. The parameters were independent because they remained significant $(p<0.05)$ in the logistic regression. The AUC of this predictive model was $0.694(95 \% \mathrm{CI}=0.640-0.731)$, and the relative ROC curve is displayed in Figure 1.

\section{Discussion}

A number of drugs useful for preventing and treating bone loss in postmenopausal patients, including bisphosphonates and selective estrogen receptor modulators (SERMs), also decrease the risk of $\mathrm{BC}(1,15)$. In particular, zoledronic acid is useful to prevent adverse skeletal events in women with 
Table I. Distribution of the main estrogen-related continuous parameters in cases and controls. Mean \pm SD and relative p-values are shown.

\begin{tabular}{lccc}
\hline Parameter & Cases & Controls & $p$-Value \\
\hline No. of patients & 297 & 297 & - \\
Age (years) & $62.0 \pm 9.1$ & $60.5 \pm 10$ & 0.057 \\
Age at menarche (years) & $12.4 \pm 1.2$ & $12.8 \pm 1.3$ & 0.0001 \\
Age at menopause (years) & $50.2 \pm 1.9$ & $49.7 \pm 1.2$ & 0.0001 \\
Interval between menarche and menopause (years) & $37.7 \pm 1.9$ & $36.9 \pm 1.9$ & 0.0001 \\
a'Age at first pregnancy (years) & $25.1 \pm 3.8$ & $24.7 \pm 3.4$ & 0.1753 \\
aParity (No. of children per woman) & $1.5 \pm 1.1$ & $1.6 \pm 1.0$ & 0.2451 \\
Serum estradiol (pmol/l) & $12.5 \pm 30.5$ & $13.0 \pm 28.9$ & 0.838 \\
bOral contraceptives use (months) & $31.2 \pm 22.4$ & $29.5 \pm 13.1$ & 0.259 \\
cHormone replacement therapy (months) & $26.3 \pm 19.6$ & $18.2 \pm 10.2$ & 0.0001 \\
\hline
\end{tabular}

In women who ahave had children, ${ }^{b}$ used oral contraceptives or ${ }^{c}$ used hormone replacement therapy, respectively.

Table II. Distribution of the main estrogen-related dichotomous parameters in cases and controls.

\begin{tabular}{|c|c|c|c|c|c|c|}
\hline Parameters & Overall (\%) & Cases $(\%)$ & Controls (\%) & OR & $95 \% \mathrm{CI}$ & $p$-Value \\
\hline No pregnancies & 16.8 & 19.2 & 14.5 & 1.40 & $0.91-2.16$ & 0.124 \\
\hline First childbearing after 30 years ${ }^{a}$ & 9.5 & 10.4 & 8.7 & 1.23 & $0.67-2.24$ & 0.507 \\
\hline No breastfeeding & 17.0 & 18.8 & 15.2 & 1.30 & $0.85-2.00$ & 0.230 \\
\hline Body mass index $\geq 25$ & 15.5 & 17.2 & 13.8 & 1.29 & $0.83-2.02$ & 0.256 \\
\hline Oral contraceptives use & 23.2 & 24.9 & 21.5 & 1.21 & $0.83-1.77$ & 0.332 \\
\hline Hormone replacement therapy & 39.6 & 44.8 & 34.3 & 1.55 & $1.11-2.16$ & 0.009 \\
\hline Osteopenia & 15.0 & 18.2 & 11.8 & 0.60 & $0.38-0.95$ & 0.028 \\
\hline Osteoporosis & 13.6 & 16.5 & 10.8 & 0.61 & $0.38-0.98$ & 0.042 \\
\hline Osteoporosis and/or osteopenia (low BMD) & 28.6 & 34.7 & 22.6 & 0.55 & $0.38-0.79$ & 0.001 \\
\hline
\end{tabular}

an women who have had children. BMD: Bone mineral density; OR: odds ratio; 95\%CI: 95\% confidence interval.

Table III. Results of the univariate analysis using the interval between menarche and menopause (MMI), hormone replacement therapy (HRT), and normal bone mineral density (BMD) as risk factors for breast cancer (95\% confidence intervals). Odds ratio (OR) and relative p-value are shown.

\begin{tabular}{lccc}
\hline Results & MMI $\geq 37.5$ years & HRT use & Normal BMD \\
\hline Sensitivity & $56.2 \%(50.6-61.9)$ & $44.8 \%(39.1-50.4)$ & $65.3 \%(59.9-70.7)$ \\
Specificity & $64.1 \%(58.7-69.5)$ & $65.7 \%(60.3-71.1)$ & $22.6 \%(17.8-27.3)$ \\
Positive predictive value & $60.7 \%(55.0-66.5)$ & $56.6 \%(50.3-62.9)$ & $45.7 \%(41.0-50.5)$ \\
Negative predictive value & $59.7 \%(54.4-65.1)$ & $54.3 \%(49.2-59.5)$ & $39.4 \%(32.1-46.8)$ \\
Positive likelihood ratio & 1.57 & 1.30 & 0.84 \\
Negative likelihood ratio & 0.68 & 0.84 & 1.54 \\
Diagnostic accuracy & $60.2 \%$ & $55.2 \%$ & $43.9 \%$ \\
OR & 2.296 & 1.550 & 1.822 \\
$p$-Value & $<0.0001$ & 0.0093 & 0.0011 \\
\hline
\end{tabular}

advanced $\mathrm{BC}$ and in those who used aromatase inhibitors and, in addition to endocrine therapy, improves both the overall survival and the disease-free survival of patients $(16$, 17). Approximately $75 \%$ of BCs occur in women aged 50 years and older, most of whom are postmenopausal and are thus exposed to the risk of progressive reduction of BMD regardless of their risk of $\mathrm{BC}$ (18). Bone mineral density, measuring the amount of bone mass per unit area $\left(\mathrm{cm}^{2}\right)$ or volume, provides accurate diagnostic criteria for evaluating bone loss, including osteoporosis, which is estimated to affect more than 22 and 10 million adults aged $\geq 50$ years in the European Union and the USA, respectively $(19,20)$. 
Table IV. Results of the multivariate analysis.

\begin{tabular}{lcrrrr}
\hline Predictive factors & T & SE & Wald & OR & $p$-Value \\
\hline MMI $>$ 37.5 years & -0.908 & 0.173 & 27.648 & 2.481 & $<0.0001$ \\
HRT use & -0.441 & 0.176 & 6.293 & 1.553 & 0.0072 \\
Osteoporosis and/or osteopenia (low BMD) & 0.743 & 0.198 & 14.033 & 0.476 & $<0.0001$ \\
\hline
\end{tabular}

MMI: Interval between menarche and menopause; HRT: hormone replacement therapy; SE: standard error; OR: odds ratio; BMD: bone mineral density.

Table V. Odds ratio (OR), 95\% confidence interval (CI) and the relative p-value using the four variables.

\begin{tabular}{llll}
\hline Parameters & OR & $95 \%$ CI & $p$-Value \\
\hline Age $>$ 55.5 years & 2.30 & $1.56-3.34$ & $<0.0001$ \\
MMI $>$ 37.5 years & 2.50 & $1.75-3.48$ & $<0.0001$ \\
HRT use & 1.79 & $1.25-2.56$ & $<0.0014$ \\
Normal BMD & 2.38 & $1.56-3.45$ & $<0.0003$ \\
\hline
\end{tabular}

MMI: Interval between menarche and menopause; HRT: hormone replacement therapy; BMD: bone mineral density.

Because luminal-type A and luminal-type B both express estrogen receptor alpha, patients with ER-positive BC can be considered a homogeneous group who are especially sensitive to the effects of circulating estrogens, which can reduce bone loss in virtually all women (21). Prolonged endogenous or exogenous estrogen exposure may stimulate the mitotic activity of the mammary cells, leading to an increased risk of $\mathrm{BC}$ development, and it has been shown that the relationship between LS-BMD and incidental BC was independent of BMI and HRT use (6). Irrespective of age, high BMD is usually considered a weak predictor of BC risk, which is mainly related to circulating estrogens but also increases in patients with low estradiol serum levels (22). This suggests that other factors, including serum testosterone and the cumulative exposure to estrogens, such as MMI and HRT, should be considered $(6,22)$. Another study hypothesized that the BMD and Gail score together could be useful predictors of $\mathrm{BC}$ risk and showed that they were independent parameters, but the gain in terms of accuracy was small $(p=0.049)(23)$.

Because in our study, low BMD, MMI and HRT were independent factors, they could be considered together in a simple predictive model for isolating women at increased risk of luminal-type BC, reaching a fair AUC. Previous studies reported AUC ranging from 0.55 to 0.67 using the Gail score alone and 0.58 and 0.67 using the Gail score together with the hip T-score or breast density, respectively

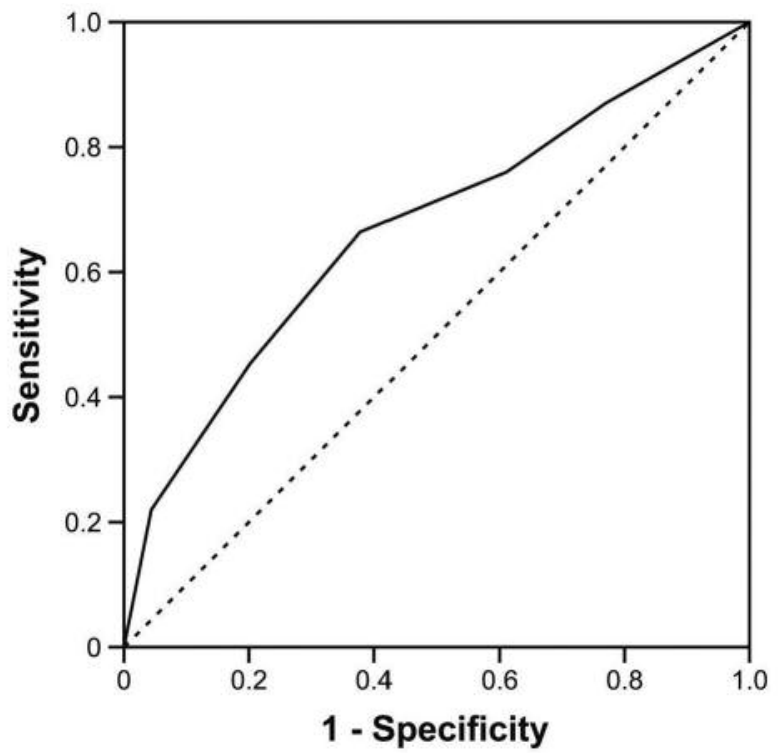

Figure 1. Receiver operating characteristic curve (ROC) and relative area under the curve (AUC) obtained using the model with the combination of all the tools (age $>55.5$ years, interval between menarche and menopause $>37.5$ years, use of hormone replacement therapy, normal bone mineral density) together.

$(23,24)$. The relationship between BMD and BC has also been questioned, but usually the study populations were not homogeneous according to the histologic type and intrinsic subtype of BC (24-26).

This study has several limitations. The main is the relatively small number of medical records evaluated $(\mathrm{N}=594)$. The reason was that we could not extend our analysis to women who had performed DXA with different bone densitometers, because the results would not be comparable. Similarly, we excluded patients with other BMD measurements, including femoral neck (hip) or phalangeal DXA, and patients who underwent quantitative ultrasound densitometry, regardless of the anatomic site of the evaluation. Another limitation is the decision to consider only the patients with luminal-type $\mathrm{BC}$. This choice was linked to 
the need to suggest a predictive model for the type of cancer mainly affected by the influence of lifelong estrogen exposure, which is the most common type of $\mathrm{BC}$, accounting for approximately $70-80 \%$ of cases $(1,27)$. However, an external validation is required before this tool can be used, because its potential predictive ability needs to be confirmed.

The main strength of our study is that it provides preliminary results for future research on the association between BMD and luminal-type BC. Additionally, our results suggest that there might be a relationship between certain traditional estrogen-related RFs, including MMI and estrogen intake, and bone mineral status of postmenopausal women.

\section{Conclusion}

Although the presence of BC itself (even in non-metastatic patients) is able to reduce BMD through various mechanisms, including the effect of chemotherapy, hormone therapy, other circulating hormones (e.g., PTH-related protein), and the premature ovarian failure, the role of estrogens remains crucial $(28,29)$. Our results suggest that age ( $>55.5$ years), MMI, HRT use, and high BMD independently lead to increased luminal-type BC occurrence. It has already been reported that lifetime estrogen exposure should be carefully evaluated in postmenopausal women, and that BMD measurement can be considered as an indicator of both bone loss, including osteopenia and osteoporosis, and BC (30).

This hypothesized predictive model is fairly accurate and could identify the patients at increased risk of developing luminal-type $\mathrm{BC}$ in a population of postmenopausal women who performed DXA, simply based on their history. However, our preliminary results should be verified by further studies with more patients.

\section{Conflicts of Interest}

The Authors declare that they have no conflicts of interest.

\section{References}

1 Lumachi F, Brunello A, Maruzzo M, Basso U and Basso SMM: Treatment of estrogen receptor-positive breast cancer. Curr Med Chem 20: 596-604, 2013.

2 Burge R, Dawson-Hughes B, Solomon DH, Wong JB, King A and Tosteson A: Incidence and economic burden of osteoporosisrelated fractures in the United States, 2005-2025. J Bone Miner Res 22: 465-475, 2007.

3 Tatalovich Z, Zhu L, Rolin A, Lewis DR, Harlan LC and Winn DM: Geographic disparities in late stage breast cancer incidence: results from eight states in the United States. Int J Health Geogr 14: 31, 2015.

4 Ferlay J, Steliarova-Foucher E, Lortet-Tieulent J, Rosso S, Coebergh JW, Comber H, Forman D and Bray F: Cancer incidence and mortality patterns in Europe: estimates for 40 countries in 2012. Eur J Cancer 49: 1374-1403, 2013.
5 Hernlund E, Svedbom A, Ivergård M, Compston J, Cooper C, Stenmark J, McCloskey EV, Jönsson B and Kanis JA: Osteoporosis in the European Union: medical management, epidemiology and economic burden. A report prepared in collaboration with the International Osteoporosis Foundation (IOF) and the European Federation of Pharmaceutical Industry Associations (EFPIA). Arch Osteoporos 8: 136, 2013.

6 van der Klift M, de Laet E, Coebergh JW, Hofman A and Pols HA: Rotterdam Study, Bone mineral density and the risk of breast cancer: The Rotterdam Study. Bone 32: 211-216, 2003.

7 Qu X, Zhang X, Qin A, Liu G, Zhai Z, Hao Y, Li H, Zhu Z and Dai K: Bone mineral density and risk of breast cancer in postmenopausal women. Breast Cancer Res Treat 138: 261-271, 2013.

8 Lumachi F, Frigo AC, Basso U, Tombolan V and Ermani M: Estrogen therapy and risk of breast cancer in postmenopausal women: a case-control study and results of a multivariate analysis. Menopause 17: 524-528, 2010.

9 Collaborative Group on Hormonal Factors in Breast Cancer: Menarche, menopause, and breast cancer risk: Individual participant meta-analysis, including 118964 women with breast cancer from 117 epidemiological studies. Lancet Oncol 13: 1141-1151, 2012.

10 Nomura SJ, Inoue-Choi M, Lazovich D, and Robien K: WCRF/AICR recommendation adherence and breast cancer incidence among postmenopausal women with and without non-modifiable risk factors. Int J Cancer 138: 2602-2615, 2016.

11 Cancer Research UK. Available at: http://www.cancer researchuk.org/about-cancer/type/breast-cancer/about/risks/ breast-cancer-protective-factors. [accessed 30 November 2017].

12 Camozzi V, Sanguin F, Albigier N, Scaroni C, Mantero F, Zaninotto M, Frigo A, Piccolo M and Luisetto G: Persistent increase of osteoprotegerin levels after cortisol normalization in patients with Cushing's syndrome. Eur J Endocrinol 162: 85-90, 2010.

13 WHO Technical Report Series. Prevention and Management of Osteoporosis. Report of a WHO Scientific Group. World Health Organization, Geneva (CH) 2003. http://apps.who.int/iris/ bitstream/10665/42841/1/WHO_TRS_921.pdf. [accessed 30 November 2017].

14 Karaguzel G and Holick MF: Diagnosis and treatment of osteopenia. Rev Endocr Metab Disord 11: 237-251, 2011.

15 Vestergaard P, Fischer L, Mele M, Mosekilde L and Christiansen P: Use of bisphosphonates and risk of breast cancer. Calcif Tissue Int 88: 255-262, 2011.

16 Camozzi V, Luisetto G, Basso SMM, Cappelletti P, Tozzoli R and Lumachi F: Treatment of chronic hypercalcemia. Med Chem 8: 556-563, 2012.

17 Gnant M, Mlineritsch B, Stoeger H, Luschin-Ebengreuth G, Knauer M, Moik M, Jakesz R, Seifert M, Taucher S, BjelicRadisic V, Balic M, Eidtmann H, Eiermann W, Steger G, Kwasny W, Dubsky P, Selim U, Fitzal F, Hochreiner G, Wette V, Sevelda P, Ploner F, Bartsch R, Fesl C and Greil R; Austrian Breast and Colorectal Cancer Study Group, Vienna, Austria: Zoledronic acid combined with adjuvant endocrine therapy of tamoxifen versus anastrozol plus ovarian function suppression in premenopausal early breast cancer: final analysis of the Austrian Breast and Colorectal Cancer Study Group Trial 12. Ann Oncol 26: 313-320, 2015. 
18 U.S. National Cancer Institute: Surveillance, Epidemiology, and End Results (SEER) Program. http://seer.cancer.gov/statfacts/ $\mathrm{html} / \mathrm{breast} . \mathrm{html}$. [accessed 20 November 2017].

19 Hernlund E, Svedbom A, Ivergård M, Compston J, Cooper C, Stenmark J, McCloskey EV, Jönsson B and Kanis JA: Osteoporosis in the European Union: medical management, epidemiology and economic burden. A report prepared in collaboration with the International Osteoporosis Foundation (IOF) and the European Federation of Pharmaceutical Industry Associations (EFPIA). Arch Osteoporos 8: 136, 2013.

20 Wright NC, Looker AC, Saag KG, Curtis JR, Delzell ES, Randall $\mathrm{S}$ and Dawson-Hughes B: The recent prevalence of osteoporosis and low bone mass in the United States based on bone mineral density at the femoral neck or lumbar spine. J Bone Miner Res 29: 2520-2526, 2014.

21 Lumachi F, Chiara GB, Foltran L and Basso SMM: Proteomics as a guide for personalized adjuvant chemotherapy in patients with early breast cancer. Cancer Genomics Proteomics 12: 385390, 2015.

22 Cauley JA, Song J, Dowsett SA, Mershon JM and Cummings SR: Risk factors for breast cancer in older women: the relative contribution of bone mineral density and other established risk factors. Breast Cancer Res Treat 102: 181-188, 2007.

23 Chen Z, Arendell L, Aickin M, Cauley J, Lewis CE, Chlebowski R, Women's Health Initiative Program, National Heart, Lung and Blood Institute, US Department of Health and Human Services: Hip bone density predicts breast cancer risk independently of Gail score: results from the Women's Health Initiative. Cancer 113: 907-915, 2008.

24 Tice JA, Cummings SR, Ziv E and Kerlikowske K: Mammographic breast density and the Gail model for breast cancer risk prediction in a screening population. Breast Cancer Res Treat 94: 115-122, 2005.
25 Stewart A, Kumar V, Torgerson DJ, Fraser WD, Gilbert FJ and Reid DM: Axial BMD, change in BMD and bone turnover do not predict breast cancer incidence in early postmenopausal women. Osteoporos Int 16: 1627-1632, 2005.

26 Trémollieres FA, Pouillès JM, Laparra J and Ribot C: Bone mineral density at menopause does not predict breast cancer incidence. Osteoporos Int 19: 1497-1504, 2008.

27 Lumachi F, Basso SM, Santeufemia DA, Bonamini M and Chiara GB: Ultrasonic dissection system technology in breast cancer: a case-control study in a large cohort of patients requiring axillary dissection. Breast Cancer Res Treat 142: 399404, 2013.

28 Hines SL, Mincey BA, Sloan JA, Thomas SP, Chottiner E, Loprinzi CL, Carlson MD, Atherton PJ, Salim M and Perez EA: Phase III randomized, placebo-controlled, double-blind trial of risedronate for the prevention of bone loss in premenopausal women undergoing chemotherapy for primary breast cancer. $\mathrm{J}$ Clin Oncol 27: 1047-1053, 2009.

29 Lumachi F and Basso SMM: Hypercalcemia: An overview of its pathology. In: Calcium: Chemistry, Analysis, Function and Effects. Preedy VC (ed.). The Royal Society of Chemistry, London, pp. 605-624, 2016.

30 Nguyen TV, Center JR and Eisman JA: Association between breast cancer and bone mineral density: the Dubbo Osteoporosis Epidemiology Study. Maturitas 36: 27-34, 2000.

Received February 17, 2018

Revised March 11, 2018

Accepted March 12, 2018 